\title{
Comparison of cobinamide to hydroxocobalamin in reversing cyanide physiologic effects in rabbits using diffuse optical spectroscopy monitoring
}

\author{
Matthew Brenner \\ Sari B. Mahon \\ University of California \\ Beckman Laser Institute \\ Laser Microbeam and Medical Program \\ Irvine, California 92612-1475 \\ and \\ University of California \\ Division of Pulmonary and Critical Care Medicine \\ Department of Medicine \\ Irvine, California 92868
}

\author{
Jangwoen Lee \\ Jae Kim \\ David Mukai \\ Seth Goodman \\ Kelly A. Kreuter \\ Rebecca Ahdout \\ University of California \\ Beckman Laser Institute \\ Laser Microbeam and Medical Program \\ Irvine, California 92612-1475
}

\section{Othman Mohammad \\ Vijay S. Sharma \\ William Blackledge}

Gerry R. Boss

University of California, San Diego

Department of Medicine

La Jolla, California 92093-0652

\begin{abstract}
Our purpose is to compare cobinamide to hydroxocobalamin in reversing cyanide $(\mathrm{CN})$-induced physiologic effects in an animal model using diffuse optical spectroscopy (DOS). Cyanide poisoning is a major threat worldwide. Cobinamide is a novel molecule that can bind two molecules of cyanide, has a much higher binding affinity than hydroxocobalamin, and is more water soluble. We investigated the ability of equimolar doses of cobinamide and hydroxocobalamin to reverse the effects of cyanide exposure in an animal model monitored continuously by DOS. Cyanide toxicity was induced in 16 New Zealand white rabbits by intravenous infusion. Animals were divided into three groups: controls $(n=5)$ received saline following cyanide, hydroxocobalamin $(N=6)$ following cyanide, and cobinamide $(N=5)$ following cyanide. Cobinamide caused significantly faster and more complete recovery of oxy- and deoxyhemoglobin concentrations in cyanide-exposed animals than hydroxocobalaminor saline-treated animals, with a recovery time constant of $13.8 \pm 7.1 \mathrm{~min}$ compared to $75.4 \pm 25.1$ and $76.4 \pm 42.7 \mathrm{~min}$, for hydroxocobalamin- and saline-treated animals, respectively $(p<0.0001)$. This study indicates that cobinamide more rapidly and completely reverses the physiologic effects of cyanide than equimolar doses of cobalamin at the dose used in this study, and $\mathrm{CN}$ effects and response can be followed noninvasively using DOS. () 2010 Society of Photo-Optical Instrumentation Engineers. [DOI: 10.1117/1.3290816]
\end{abstract}

Keywords: spectroscopy; optical systems; photodetectors.

Paper 09200R received May 18, 2009; revised manuscript received Sep. 26, 2009; accepted for publication Nov. 11, 2009; published online Jan. 13, 2010.

\section{Introduction}

Cyanide $(\mathrm{CN})$ poisoning can occur from a diverse range of civilian and military exposures. ${ }^{1}$ Sources include medications such as nitroprusside, accidental ingestion of naturally occurring cyanogenic compounds, and industrial accidents involving fumigants, gold mining and electroplating solvents, and other manufacturing by-products. ${ }^{2}$ In addition, smoke from residential or industrial fires contains a complex mixture of gases, including hydrogen cyanide. ${ }^{3-6}$ Military and civilian exposures to cyanide may occur through acts of terrorism and war, including both the direct use of cyanide or cyanogenic compounds, as well as indirectly as a result of fire-causing incendiary devices and explosives. ${ }^{1,7-9}$

Current treatments for cyanide exposure include three general classes of agents: methemoglobin generators (sodium nitrite, amyl nitrite, and dimethyl aminophenol), sulphur donors (sodium thiosulfate and glutathione), and direct binding

Address correspondence to: Dr. Matthew Brenner, University of California, Beckman Laser Institute, Laser Microbeam and Medical Program, Irvine, California 92612-1475. Tel: 714-456-5150; Fax: 714456 8349; E-mail: mbrenner@uci.edu agents (hydroxocobalamin and dicobalt edetate). ${ }^{1,2}$ Each of these treatment modalities has advantages and limitations. ${ }^{1,2}$ Nitrites are relatively contraindicated in smoke inhalation victims, because the methemoglobin they produce, when combined with carboxyhemoglobin from carbon monoxide, can significantly reduce the oxygen carrying capacity of blood. ${ }^{2,10}$ Sulfur donors can be used for smoke inhalation victims, but they are slow in onset of activity. Dicobalt ethelynediaminetetraacetic acid (EDTA) can cause hypotension and risks of toxicity from cobalt to the extent that it should be administered only in confirmed cases of cyanide poisoning. ${ }^{2}$ Hydroxocobalamin, which was recently approved for cyanide poisoning by the U.S. Food and Drug Administration, can also be used in the presence of carbon monoxide, but it needs to be given in relatively large volumes. ${ }^{11,12}$

New methods for the timely recognition and diagnosis of possible cyanide poisoning, and for determining the effectiveness of various treatment regimes, are essential to improving the likelihood of successful outcomes in military and civilian populations. ${ }^{13,14}$ Cyanide ion binds to oxidized iron in cyto-

$1083-3668 / 2010 / 15(1) / 017001 / 8 / \$ 25.00$ ○ 2010 SPIE 
chrome oxidase, preventing its reduction, and thereby blocking electron transport and interrupting cellular respiration. ${ }^{1,2,15-18}$ Tissues are unable to extract oxygen from hemoglobin, and circulating blood becomes more saturated with oxygen. ${ }^{19}$ In this study, we used diffuse optical spectroscopy (DOS) to rapidly and continuously monitor cyanide toxicity and therapeutic responses. DOS is able to simultaneously measure near-infrared (NIR) tissue optical scattering and absorption, resulting in accurate, quantitative measures of tissue oxy-, met-, carboxy-, and deoxyhemoglobin. ${ }^{20-26}$ The measured hemoglobin represents that contained within arterial, capillary, and venous vessels, and the volume of the vascular compartment as a fraction of the total tissue volume. Thus, DOS has the potential to assess the extent of physiologic changes induced by cyanide exposure and quantitatively compare the effectiveness of various treatment regimens.

Cobinamide is a novel agent we are investigating for treating cyanide poisoning ${ }^{27-29}$ that appears to have some advantages over current treatments, including hydroxocobalamin. Cobinamide is the penultimate precursor in the biosynthesis of cobalamin, lacking the dimethyl-benzimidazole ribonucleotide tail coordinated to the cobalt atom in the lower axial position (Fig. 1). Whereas hydroxocobalamin has only an upper ligand binding site, cobinamide has both an upper and a lower ligand binding site. The dimethylbenzimidazole group exerts a negative trans-effect on the upper binding site, thereby reducing hydroxocobalamin's affinity for ligands. ${ }^{27}$ The combined effect is that each cobinamide molecule can bind two cyanide molecules and that cobinamide has a much greater affinity for cyanide than hydroxocobalamin, with a $K_{A}$ overall of $\approx 10^{22} \mathrm{M}^{-1}$ (binding affinity for first cyanide ion is $10^{14} \mathrm{M}^{-1}$ and for second ion is $\left.10^{8} \mathrm{M}^{-1}\right){ }^{30}$ This suggests that cobinamide should be a more effective cyanide detoxifying agent than hydroxocobalamin (which has a $K_{A}$ of $10^{12} \mathrm{M}^{-1}$ for cyanide), as we have observed previously. ${ }^{25}$ In aqueous solution, cobinamide exists as aquohydroxocobinamide, which is at least five times more water soluble than hydroxocobalamin. The combination of cobinamide's high binding affinity for cyanide, binding of two cyanide molecules, and relatively high water solubility suggest it could eventually be formulated for administration in concentrated solutions for intramuscular injection (raising the possibility of eventual development of a treatment modality for mass casualty cyanide exposure, which is currently unavailable). For these reasons, we investigated the effects of administration of intravenous cobinamide compared to hydroxocobalamin in reversing cyanide exposure effects in an animal model. ${ }^{20}$

We previously showed that DOS detects pathophysiologic events occurring in cyanide-poisoned animals. ${ }^{20}$ In the current study, we compare cobinamide to hydroxocobalamin in reversing cyanide effects using DOS, as well as measuring standard red blood cell (RBC) cyanide concentrations.

\section{Methods}

The methods for cyanide induction and DOS monitoring in rabbits have been previously described ${ }^{20}$ and are summarized here. (a) Cobalamin
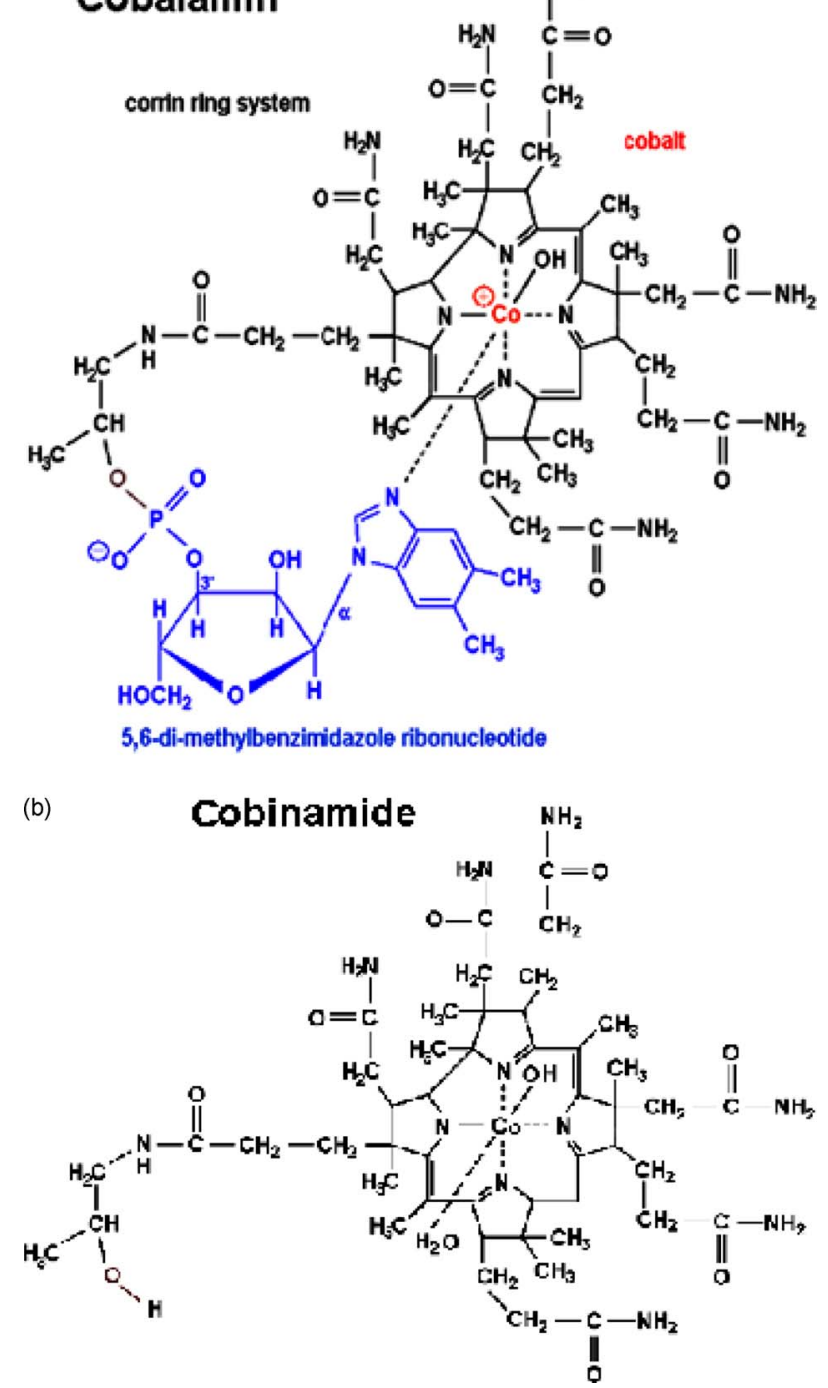

Fig. 1 Comparison of the molecular structure of (a) hydroxocobalamin to (b) cobinamide (lower panel). Cobinamide lacks the dimethyl-benzimidazole ribonucleotide tail coordinated to the cobalt atom in the lower axial position. Whereas cobalamin has only an upper ligand binding site, cobinamide has both an upper and a lower ligand binding site. The dimethylbenzimidazole group on cobalamin has a negative trans-effect on the upper binding site, thereby reducing cobalamin's affinity for ligands. ${ }^{11}$ The combined effect is that each cobinamide molecule can bind two cyanide molecules and that cobinamide has a much greater affinity for cyanide than cobalamin.

\subsection{General Preparation}

The protocol was reviewed and approved by the University of California Irvine (UCI) Institutional Animal Care and Use Committee (IACUC). Sixteen pathogen-free New Zealand white rabbits (Western Oregon Rabbit Supply), weighing 3.5 to $4.5 \mathrm{~kg}$ were used in this study: five received cyanide followed by normal saline, five received cyanide followed by hydroxocobalamin, and five received cyanide followed by cobinamide. Animals were anesthetized with an intramuscular injection of Ketamine $\mathrm{HCl} 50 \mathrm{mg} / \mathrm{kg}$ (Ketaject, Phoenix Pharmaceutical, Inc., St. Joseph, Michigan) and Xylazine $5 \mathrm{mg} / \mathrm{kg}$ (Anased, Lloyed Laboratories, Shenandoah, Iowa) 
using a 23-gauge, 5/8-in needle. After the injection, a 23gauge, 1 -in catheter was placed in the animal's marginal ear vein to administer continuous intravenous anesthesia. The depth of anesthesia was evaluated by monitoring the animals' physical reflexes and heart rate. Torbutrol $0.5 \mathrm{mg} / \mathrm{kg}$, was given subcutaneously, and then the animals were intubated with a 3.0 cuffed endotracheal tube secured by a gauze tie; they were mechanically ventilated (dual-phase control respirator, Model 32A4BEPM-5R, Harvard Apparatus, Chicago) at a rate of 32 respirations per min, a tidal volume of $50 \mathrm{cc}$, and $\mathrm{FiO}_{2}$ of $100 \%$. A pulse oximeter (Biox 3700 Pulse Oximeter, Ohmeda, Boulder, Colorado) with a probe was placed on the tongue, to measure $\mathrm{SpO}_{2}$ and heart rate.

\subsection{Systemic Arterial Blood Pressure and Blood Sampling}

Femoral arterial and venous cutdowns were performed for central line placement to collect blood samples and measure systemic pressure. A 3-in incision was made with a \#10 scalpel blade on the shaved left hind leg of the animals, and blunt dissection was used to isolate the vein and artery. A 12-in, 18-gauge catheter (C-PMA-400-FA, Cook, Inc., Bloomington, Indiana) was inserted into each, and a three-way stopcock was connected. A calibrated pressure transducer (TSD104A Transducer and MP100 WSW System, Biopac Systems, Inc., Santa Barbara, California) was connected to the end of the arterial line. ${ }^{28,31}$ On completion of the experiment, the animals were euthanized with an intravenous injection of Euthasol $(1.0 \mathrm{cc}$, Euthasol, Virbac AH, Inc., Fort Worth, Texas; 390 mg pentobarbital sodium; $50 \mathrm{mg}$ phenytoin sodium $/ \mathrm{ml}$ ) administered through the marginal ear vein.

\subsection{Cyanide Infusion and Administration of Antidotes}

Sodium cyanide, $10 \mathrm{mg}$ in $60 \mathrm{cc}$ normal saline, was infused intravenously at a rate of $1 \mathrm{cc} / \mathrm{min}$ for $60 \mathrm{~min}$. At the end of the cyanide infusion, $5 \mathrm{cc}$ of normal saline (control animals) or 0.0816 millimoles of cobinamide or hydroxocobalamin dissolved in $5 \mathrm{cc}$ of normal saline were infused over $30 \mathrm{~s}$. Cobinamide was prepared from hydroxocobalamin by base hydrolysis at $\mathrm{pH} 9.5$ using cerium hydroxide. ${ }^{32}$ The cobinamide product was separated from unreacted hydroxocobalamin on a weak cation exchange column eluted with a $\mathrm{NaCl}$ gradient. It was desalted on a $\mathrm{C}_{18}$ reverse-phase resin column, concentrated on a rotary evaporator, and lyophilized to a solid state. At neutral pH, cobinamide exists as aquohydroxocobinamide, which is referred to as "cobinamide" throughout the text.

\subsection{DOS Measurements}

DOS measurements were obtained through a fiber-optic probe with a diode light emitter and detector at a fixed distance $(10 \mathrm{~mm})$ from the source fiber, which was placed on the shaved surface of the right inner thigh of the animal. The broadband DOS system we constructed combines multifrequency domain (FD) photon migration with time-independent near-infrared spectroscopy to accurately measure bulk tissue absorption and scattering spectra. ${ }^{20-22,24-26}$ It employs six laser diodes at discrete wavelengths $(661,681,783,805,823$, and $850 \mathrm{~nm}$ ), and a fiber-coupled avalanche photo diode (APD) detector (Hamamatsu high-speed APD module C5658,
Bridgewater, New Jersey) for the frequency-domain measurements. The APD detects the intensity-modulated diffuse reflectance signal at modulation frequencies between 50 and $300 \mathrm{MHz}$ after propagation through the tissue. Absorption and reduced scattering coefficients are measured directly at each of the six laser diode wavelengths using frequency-dependent phase and amplitude data. Reduced scattering coefficients are calculated as a function of wavelength throughout the near-infrared region by fitting a power-law to six reduced scattering coefficients. Steady-state acquisition was a broadband reflectance measurement from 650 to $1000 \mathrm{~nm}$ that follows frequency-domain measurements using a tungsten-halogen light source (Ocean Optics HL-2000, Dunedin, Florida) and a spectrometer (BWTEK BTC611E, Newark, Delaware). Intensity of the steady-state reflectance measurements are calibrated to the frequencydomain values of absorption and scattering to establish the absolute reflectance intensity. ${ }^{20,24,25}$ Tissue concentrations of oxy- and deoxyhemoglobin are calculated by a linear leastsquares fit of the wavelength-dependent extinction coefficient spectra of each chromophore. We used oxy- and deoxyhemoglobin absorption spectra reported by Zijlistra et al. ${ }^{33}$ for subsequent fitting and analysis. The wavelengths measured by DOS were chosen exclusively above $650 \mathrm{~nm}$ in order to avoid any potential interference by cobinamide or hydroxocobalamin absorbance with oxy- and deoxy-hemoglobin measurements. DOS measurements were taken continuously throughout the experiments.

\subsection{Measurement of Red Blood Cell (RBC) Cyanide Concentration}

Cyanide in blood is bound almost exclusively to ferric(met)hemoglobin in RBCs; thus, blood cyanide can be measured by separating RBCs from plasma and acidifying the $\mathrm{RBCs}$ to release cyanide as hydrogen cyanide $(\mathrm{HCN})$ gas. $^{34}$ Blood was drawn for cyanide levels at baseline; 5 min prior to completing the cyanide infusion; at the end of the cyanide infusion; and at 2.5, 5, 10, 15, 30, 45, and 60 min after administering saline, cobinamide, or hydroxocobalamin. The blood was immediately cooled to $4^{\circ} \mathrm{C}$ and centrifuged, and the plasma and $\mathrm{RBC}$ fractions were separated. Samples were kept at $4{ }^{\circ} \mathrm{C}$ and analyzed within $48 \mathrm{~h}$. The RBCs were lysed in ice-cold water, and the lysates were placed into glass tubes sealed with stoppers holding plastic center wells (Kontes Glass Co., Vineland, New Jersey) containing 0.1 M NaOH. A volume of $10 \%$ trichloroacetic acid equal to the lysate was injected through the stopper into the tubes, and the tubes were shaken at $37^{\circ} \mathrm{C}$ for $75 \mathrm{~min}$. After cooling to room temperature, cyanide trapped in the $\mathrm{NaOH}$ was measured by following its reaction with p-nitrobenzaldehyde and o-dinitrobenzene at $560 \mathrm{~nm}$ (Ref. 31). Concentrations were determined from standard curves using freshly prepared potassium cyanide $(\mathrm{KCN})$ dissolved in $0.1 \mathrm{M} \mathrm{NaOH}$. Duplicate samples showed $<15 \%$ variation.

\subsection{Statistical Methods}

Baseline statistical parameters across groups were compared using analysis of variance. Response to treatment across the three groups was compared using analysis of variance with repeated measures for cyanide level comparisons. Time con- 
stants for DOS changes in tissue hemoglobin oxygenation were compared using analysis of variance. A two-tailed $p$-value $<0.05$ was considered significant. All data was analyzed using a standard statistical package (Systat-12, Systat Software, Inc., Chicago).

\section{Results}

\subsection{Assessment of Oxy- and Deoxyhemoglobin Concentrations}

Physiologic effects of cyanide poisoning were readily seen, as evidenced by a marked increase in tissue oxyhemoglobin and decrease in deoxyhemoglobin concentrations within minutes of starting the cyanide infusion (Figs. 2(a)-2(c) show representative animals). After stopping the cyanide infusion, the oxy- and deoxyhemoglobin concentrations slowly returned towards baseline in the control group, although they never fully recovered to baseline values, even after 90 min [Fig. 2(a)]. In animals that received hydroxocobalamin, oxy- and deoxyhemoglobin concentrations slowly returned to baseline values over about $65 \mathrm{~min}$ [Fig. 2(b)]. This is in contrast to cobinamide-treated animals in which the oxy- and deoxyhemoglobin concentrations returned to baseline values within 10 min of cobinamide infusion [Fig. 2(b)]. The data for all animals in each group were plotted as fractional change in deoxy- [Fig. 3(a)] and oxyhemoglobin [Fig. 3(b)] concentrations to calculate rates of reversal. Reversal rates were not significantly different between hydroxocobalamin-at the dose used in this study-and saline-treated animals ( $p$ $>0.95)$, whereas reversal rates were significantly faster in the cobinamide-treated than saline-treated animals $(p<0.05)$.

As may be seen in Figs. 2 and 3, cobinamide induced an apparent "overshoot" of oxy- and deoxyhemoglobin concentrations beyond baseline pre-cyanide infusion values. This likely is a result of cobinamide binding of nitric oxide (see discussion).

\subsection{Time Constants for Recovery of Deoxyhemoglobin Concentration}

The time constant for recovery of deoxygenated hemoglobin to baseline, pre-cyanide exposure levels is calculated as $\sim 1-\exp (-t / \tau)$, where $\tau$ is a time constant. $\tau$ from the data fittings are listed below in Table 1, showing the effective time to recovery (to $66.6 \%$ of baseline) using previously described methods. ${ }^{35}$ Using this formula, the time constant for recovery of deoxygenated hemoglobin toward baseline was $13.8 \mathrm{~min}$ for the cobinamide-treated animals, compared to approximately $75 \mathrm{~min}$ for the control and hydroxocobalamin-treated animals $(p<0.0001)$.

In the crucial first 10 min following cyanide poisoning, the slope of recovery of deoxygenated hemoglobin for the cobinamide-treated group was more than five times greater than for hydroxocobalamin and controls (Table 2). There was no difference in recovery rates between control and hydroxocobalamin-treated animals $(p>0.15)$.

\subsection{Assessment of Total Tissue Hemoglobin}

Total tissue hemoglobin concentrations before and following recovery from cyanide infusion were the same in all groups (data not shown).

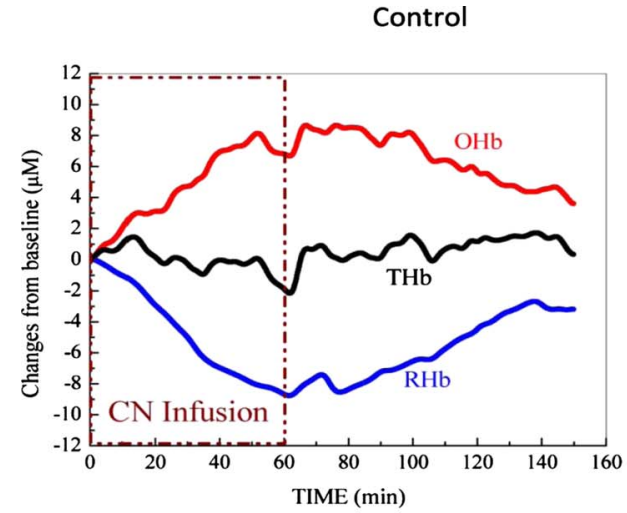

(a)

Hydroxocobalamin

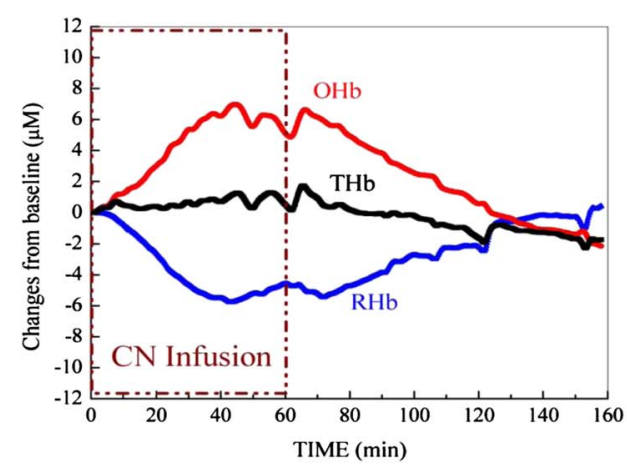

(b)

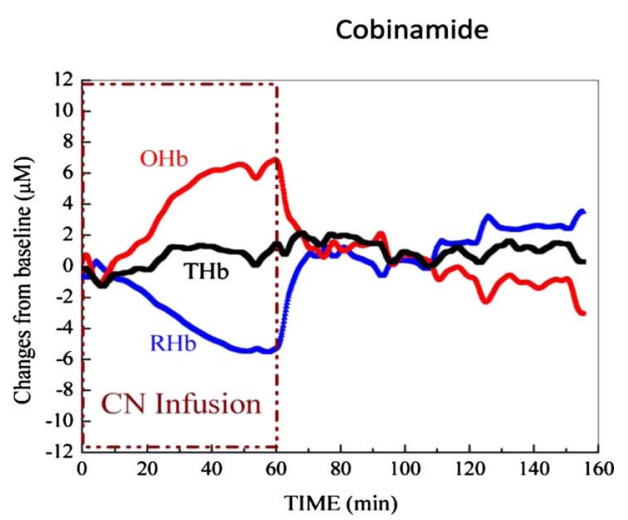

(c)

Fig. 2 Effects of cobinamide and hydroxocobalamin on tissue oxyhemoglobin, deoxyhemoglobin, and total hemoglobin concentrations in representative cyanide-poisoned rabbits. Changes in tissue oxyhemoglobin $(\mathrm{OHb})$, deoxyhemoglobin $(\mathrm{RHb})$, and total hemoglobin $(\mathrm{THb})$ as measured by DOS are shown during and following cyanide infusion in individual rabbits. The decrease in deoxyhemoglobin concentration during $60 \mathrm{~min}$ of cyanide infusion is due to the inability of tissues to remove oxygen from circulating blood, leaving more hemoglobin in the oxygenated state. At 60 min, when the cyanide infusion was stopped, the animals received either 5 cc of saline (a), 5 cc of $16.3 \mathrm{mM}$ cobalamin in saline (b), or $5 \mathrm{cc}$ of $16.3 \mathrm{mM}$ cobinamide in saline (c). In the control animal, oxy- and deoxyhemoglobin concentrations gradually returned toward baseline after the cyanide infusion was discontinued but never fully returned to preinfusion values. In the hydroxocobalamin-treated animal, oxy- and deoxyhemoglobin concentrations returned to baseline at $\sim 130 \mathrm{~min}$, i.e., $70 \mathrm{~min}$ following drug administration. In contrast, oxy- and deoxyhemoglobin concentrations returned to baseline within $10 \mathrm{~min}$ following cobinamide administration. 
Brenner et al.: Comparison of cobinamide to hydroxocobalamin in reversing cyanide physiologic effects in rabbits...
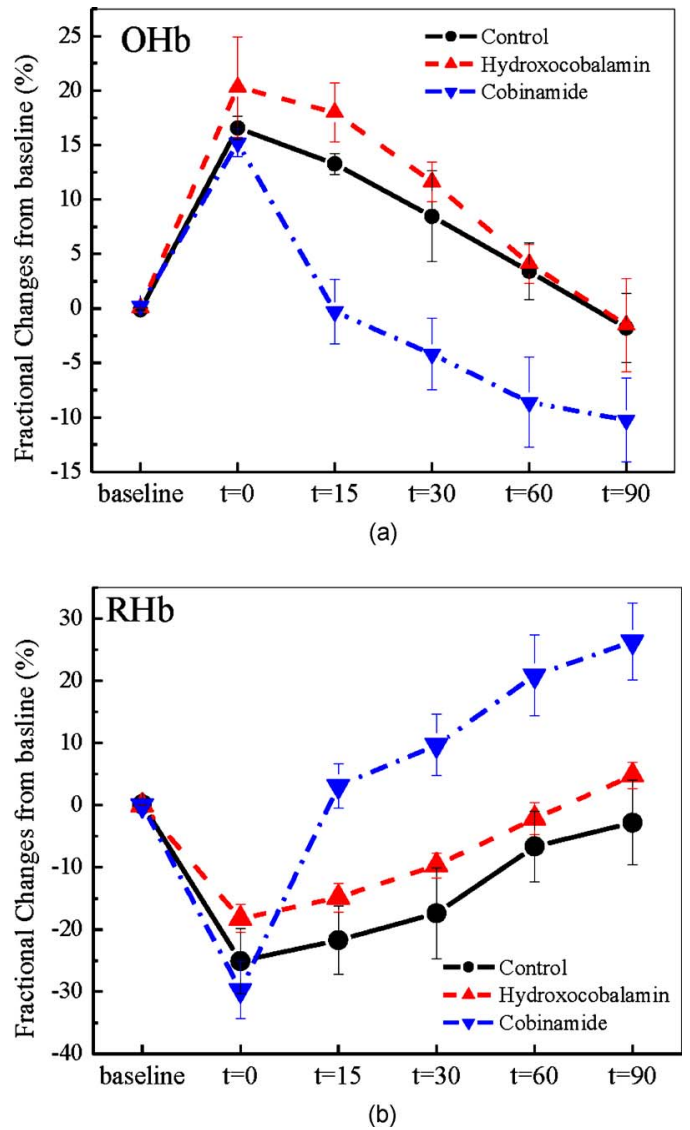

Fig. 3 Effect of cobinamide and hydroxocobalamin on tissue oxy- and deoxyhemoglobin concentrations in all cyanide-poisoned rabbits. The mean and standard error of percent change in (a) oxyhemoglobin $(\mathrm{OHb})$ and (b) deoxyhemoglobin $(\mathrm{RHb})$ concentrations from baseline are shown during cyanide infusion and treatment for control animals and for hydroxocobalamin-treated (IV OHCob) or cobinamide-treated (IV Cob) animals. (a) Tissue deoxyhemoglobin concentrations rise rapidly from their levels at the end of the cyanide infusion in cobinamide-treated animals compared to controls and hydroxocobalamin animal groups. (b) Tissue oxyhemoglobin concentrations fall significantly faster from their peak value at the end of cyanide infusion following treatment with cobinamide compared to hydroxocobalamin or controls.

\subsection{Assessment of Blood Cyanide Concentrations}

Blood cyanide concentrations were similar at baseline and post-cyanide infusion in all three treatment groups, rising from a mean of $1.8 \pm 1.8$ to $221 \pm 10.5 \mu \mathrm{M}$ (SEM) over the 60 -min infusion period (mean \pm SEM). There was no differ-

Table 1 Time constant values for return of deoxyhemoglobin concentration toward baseline values: recovery time curves are fitted by using an exponential model and shown as mean \pm standard deviation. Analysis was performed using ANOVA assuming unequal variances; in comparing cobinamide to controls, $p<0.0001$ for the deoxyhemoglobin time constant.

\begin{tabular}{lccc} 
Time constant $\backslash$ group & Control & Hydroxocobalamin & Cobinamide \\
\hline $\begin{array}{c}\text { Deoxyhemoglobin } \\
\text { recovery }(\mathrm{min})\end{array}$ & $76.4 \pm 42.7$ & $75.4 \pm 25.1$ & $13.8 \pm 7.1$ \\
\hline
\end{tabular}

Table 2 Initial $10 \mathrm{~min}$ of hemodynamic recovery slope of percent change deoxyhemoglobin concentration back toward normal in muscle region after hydroxocobalamin or cobinamide administration shown as mean \pm standard deviation.

\begin{tabular}{cccc}
\hline Slope Cgroup & Control & Hydroxocobalamin & Cobinamide \\
\hline $\begin{array}{c}\text { Deoxyhemoglobin } \\
\text { recovery }\end{array}$ & $0.34 \pm 0.12$ & $0.5 \pm 0.25$ & $3.0 \pm 0.11$ \\
(percentage/min) & & & \\
\hline
\end{tabular}

ence in baseline cyanide levels or peak cyanide levels following cyanide infusion in the three animal groups $(p=0.30$ ANOVA for differences across groups).

The cyanide concentrations returned more rapidly toward baseline values in cobinamide-treated animals than in hydroxocobalamin- or saline-treated animals (Fig. 4; $p$ $<0.01$ ANOVA with repeated measures). Thus, at $30 \mathrm{~min}$ following completion of the cyanide infusion, blood cyanide concentrations had decreased to $38.2 \pm 8 \%$ of peak values in the cobinamide-treated animals, compared to $82.4 \pm 8 \%$ and $92.0 \pm 7.41 \%$ of peak values in saline- and hydroxocobalamin-treated animals, respectively. By comparing Fig. 4 with Figs. 2 and 3, it can be noted that oxy- and deoxyhemoglobin concentrations reversed more quickly than blood cyanide levels in the cobinamide-treated group.

\section{Discussion}

Cyanide poisoning presents an ongoing diagnostic and therapeutic challenge. The onset of cyanide poisoning is rapid and difficult to recognize. We investigated the ability of intravenous cobinamide to reverse the physiologic effects of cyanide exposure compared to hydroxocobalamin, an approved cyanide antidote. At equimolar doses of cobinamide and hydroxocobalamin (at a dose calculated to bind the administered

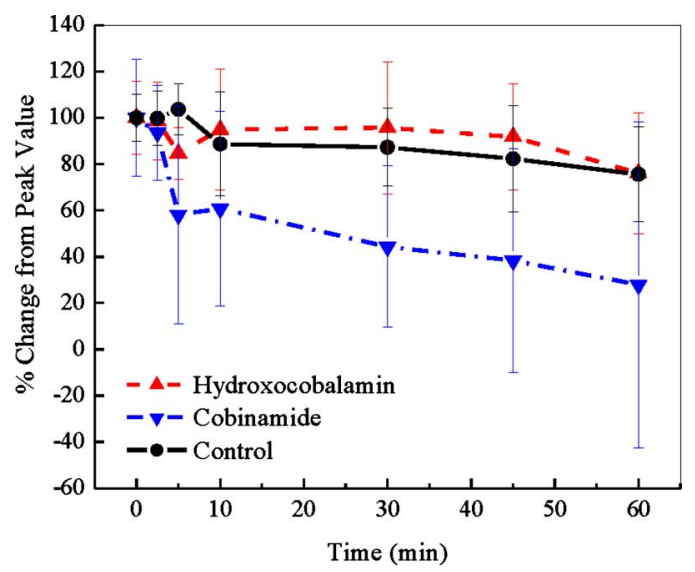

Fig. 4 RBC cyanide concentrations. The concentration of cyanide in RBCs is shown at the end of the cyanide infusion (zero time) and for $60 \mathrm{~min}$ thereafter. At zero time, the animals received saline (controls), hydroxocobalamin, or cobinamide. Values are expressed as percent of the peak cyanide levels achieved at the completion of cyanide infusion and shown as mean and standardized error. Cobinamide-treated animals had significantly faster reduction in blood cyanide levels compared to hydroxocobalamin and control groups. 
molar dose of cyanide by cobinamide), cobinamide reversed the pathophysiological effects of cyanide much more rapidly than hydroxocobalamin, as measured by changes in tissue oxy- and deoxyhemoglobin concentrations and blood cyanide concentrations.

There are two likely reasons for the increased effectiveness of cobinamide to reverse cyanide toxicity compared to hydroxocobalamin. First, cobinamide has a much higher affinity for cyanide than hydroxocobalamin. Second, cobinamide has two ligand binding sites compared to a single binding site on hydroxocobalamin. Thus, at equimolar concentrations, cobinamide would be expected to bind more cyanide molecules and show increased effectiveness. The rate of recovery of oxy- and deoxyhemoglobin concentrations were no different in hydroxocobalamin-treated animals than in controls. At higher hydroxocobalamin concentrations (such as double the hydroxocobalamin concentration, where the dose would be the molar equivalent of the cyanide dose), reversal of cyanide effects would likely have occurred, since hydroxocobalamin is an effective treatment for cyanide toxicity. However, our purpose was to compare cobinamide and hydroxocobalamin at equimolar doses, rather than repeat a demonstration of the effectiveness of hydroxocobalamin. Thus, we selected a drug dose designed to elucidate differences between the two drugs.

In addition to cyanide, cobinamide is capable of reacting with nitric oxide (NO). The binding constant to cobinamide is substantially lower for NO than it is for cyanide $\left(\approx 10^{22} \mathrm{M}^{-1}\right.$ for cyanide versus $\approx 10^{10} \mathrm{M}^{-1}$ for NO). ${ }^{27,30}$ Thus, cobinamide preferentially binds cyanide, and we showed previously that cobinamide can neutralize nitroprusside-released cyanide without having any effect on nitroprusside-released $\mathrm{NO}^{29}$ However, when given in excess of available cyanide, cobinamide could potentially induce vasoconstriction and increase blood pressure, as has occurred with hydroxocobalamin. ${ }^{36}$ It is possible that cobinamide's reaction with NO was responsible for the observed recovery phase "overshoot" in oxy- and deoxyhemoglobin concentrations observed in some cobinamidetreated animals.

Of interest is the variation in correlation between reversal of the physiological effects of cyanide toxicity as measured by oxy- and deoxyhemoglobin concentrations and blood cyanide levels. The oxy- and deoxyhemoglobin changes measured by DOS do show what appears to be complete reversal of cyanide exposure by both cobinamide and hydroxocobalamin, as measured by this limited outcome variable. Blood cyanide levels, especially following cobinamide treatment, are significantly decreased but do not return to baseline levels within the experimental time frame. Cyanide is an extraordinarily complex molecule in terms of its toxicity and detoxification. The nonlinear response behavior and nonlinear relationship between cytochrome inhibition and cellular oxidative metabolism has been described in numerous studies. Extensive studies of dose response (in terms of cyanide doses as well as treatment agent doses), timing of treatment administration, and formal modeling of interactions will be critical to fully defining potential benefits and limitations of cyanide treatment agents such as cobinamide. This was a sacrifice study, and the animals were not awakened. No neurological testing or other investigations of toxicity, organ dysfunction, or other important parameters were done that would be needed before any claims of "complete cyanide reversal" can be made with certainty.

Rapid diagnosis of cyanide toxicity and continuous, quantitative assessment of response to treatment is essential for optimization of cyanide therapy and comparison of effectiveness of treatment agents. Cyanide itself is not easily detected by rapid assay, either in blood samples or through noninvasive monitoring. Furthermore, blood cyanide levels do not correlate closely with the degree of toxicity for a number of welldescribed reasons. ${ }^{14,37-40}$ Therefore, methods for assessing the physiologic effects of cyanide may be more clinically relevant indicators of cyanide effects and response to therapy. The DOS system we used may provide a much-needed ability for noninvasive determination of the impact of cyanide on physiologic function. ${ }^{20}$ The DOS system used in this study could be miniaturized to a small, hand-held, field-deployable device that could be used for rapid identification and monitoring of cyanide poisoning in the field or in hospital settings.

Hydroxocobalamin absorbs in the $600-\mathrm{nm}$ region, and we have shown that it can interfere with measurements of oxyand deoxyhemoglobin concentration by standard cooximetry. ${ }^{41}$ The absorption in the $600-\mathrm{nm}$ region by hydroxocobalamin must be taken into consideration in DOS measurements as well. Therefore, in this study, we utilized laser emitting diode frequencies greater than $650 \mathrm{~nm}$ and analyzed the continuous absorption spectra greater than $654 \mathrm{~nm}$ for calculations in order to eliminate possible hydroxocobalamin-induced artifacts. In this manner, DOS has capabilities for assessing the physiologic response to therapeutic interventions in cyanide toxicity and therefore may be an ideal tool for comparing various therapeutic regimens.

In addition to the greater cyanide binding efficiency of cobinamide compared to hydroxocobalamin, cobinamide is more soluble in aqueous medium. Therefore, cobinamide can be administered rapidly in small volumes, e.g., over less than $30 \mathrm{~s}$ intravenously. As approved, hydroxocobalamin should be administered over 15 min (Ref. 42), which may be an issue in acutely cyanide-toxic patients. Furthermore, physical incompatibility (particle formation) was observed in mixtures of hydroxocobalamin with diazepam, dobutamine, dopamine, fentanyl, nitroglycerin, pentobarbital, propofol, and thiopental. ${ }^{42}$ We have not assessed these compatibility issues with cobinamide. However, the ability to rapidly administer cobinamide may alleviate compatibility concerns regarding more prolonged intravenous infusion of hydroxocobalamin.

There are a number of limitations to this study. All animals were anesthetized for comfort and safety assurances in compliance with animal welfare regulations. This could potentially have interfered with our measures of oxy- and deoxyhemoglobin concentrations, but the effect should have been the same across the three treatment groups. This is a sublethal cyanide animal model. All animals survived, even in the control group that did not receive any treatment following cyanide infusion. Therefore, we did not demonstrate whether cobinamide is capable of preventing cyanide-induced mortality from this study. Future investigations will be required for survival efficacy determination. We used a suboptimal hydroxocobalamin dose to compare equal molar effectiveness to cobinamide. To determine whether hydroxocobalamin could be equally, or potentially more, effective than cobinamide when 
both drugs are used at optimal doses will require future investigation. There are other potential advantages of cobinamide over hydroxocobalamin, even if both drugs are equally effective when administered intravenously. In particular, cobinamide has the potential to be given intramuscularly, and if developed as an intramuscular therapy, would be of significant advantage for potential treatment of mass casualty cyanide exposure victims. Specific DOS limitations include the fact that DOS measures average tissue constituents to a depth of $\sim 4$ to $8 \mathrm{~mm}$ (at the source detector separation of $10 \mathrm{~mm}$ used in the studies). Deeper tissue effects cannot be assessed using the current study design.

The ability of DOS to identify cyanide toxic victims when baseline oxy/deoxyhemoglobin ratios are not available has not been established. Thus, while the ability to track changes occurring during cyanide exposure and reversal has been clearly shown, the ability to obtain an initial definitive diagnosis has not been demonstrated. Furthermore, other potential optically interfering agents or medical conditions that might alter oxyhemoglobin levels could affect the ability to diagnose and monitor cyanide toxicity, and include commonly encountered combined carbon monoxide cyanide toxicity states. DOS capabilities in these scenarios will need to be investigated as well. In addition, regional and organ specific toxicity issues for cyanide were not addressed in this study. Future studies using lethal doses and other modes of administration are indicated to determine the possibility of developing mass casualty cyanide $(\mathrm{CN})$ exposure treatments.

In conclusion, this study demonstrates the effectiveness of cobinamide in reversing the physiologic effects of cyanide on oxy- and deoxyhemoglobin. The high binding capacity of cobinamide for cyanide and its relatively high solubility in aqueous solution may provide advantages in drug delivery and ultimately in treatment effectiveness. This study also demonstrates the capabilities of DOS to noninvasively assess and continuously monitor the physiologic effects of cyanide in vivo. This technology may provide an ideal method for rapid field evaluation of cyanide exposure effects and optimization of therapeutic regimens and may provide a platform for quantitative comparisons among various potential cyanide treatment regimens.

\section{Acknowledgments}

We would like to thank Seth Goodman for his help in the preparation and submission of this manuscript and Tanya Burney for her technical assistance with the investigations performed in this study. Supported by funding sources AF-955008-1-0384, LAMMP NIH No. P41RR01192, NIH No. 1U54NS063718-01, NIH No. U01-NS058030.

\section{References}

1. S. I. Baskin and T. G. Brewer, "Medical aspects of chemical and biological warfare," Chapter 10 in Textbook of Military Medicine Part I, Warfare, Weaponry, and the Casualty, F. R. Sidell, E. T. Takafuji, and D. R. Franz, Eds., pp. 272-286, Borden Institute, Walter Reed Army Medical Center; Office of the Surgeon General, U.S. Army; U.S. Army Medical Dept. Center and School; U.S. Army Medical Research and Material Command; Uniformed Services University of the Health Sciences, Washington, DC (1997).

2. T. F. Cummings, "The treatment of cyanide poisoning," Occup. Med. (Oxf) 54(2), 82-85 (2004)
3. A. Strickland, R. Y. Wang, R. S. Hoffman, and L. R. Goldfrank, "Blood cyanide concentrations after smoke inhalation," N. Engl. J. Med. 326(20), 1362 (1992).

4. K. Riddle, "Hydrogen cyanide: fire smoke's silent killer," J. Emerg. Med. Serv. (JEMS) 29(8), suppl. 5 (2004).

5. D. J. Barillo, R. Goode, and V. Esch, "Cyanide poisoning in victims of fire: analysis of 364 cases and review of the literature," J. Burn Care Rehabil. 15(1), 46-57 (1994).

6. R. Alcorta, "Smoke inhalation and acute cyanide poisoning. Hydrogen cyanide poisoning proves increasingly common in smokeinhalation victims," J. Emerg. Med. Serv. (JEMS) 29(8), suppl. 6-15, quiz suppl. 16-17 (2004).

7. C. O. Martin and H. P. Adams Jr., "Neurological aspects of biological and chemical terrorism: a review for neurologists," Arch. Neurol. 60(1), 21-25 (2003).

8. R. Gracia and G. Shepherd, "Cyanide poisoning and its treatment," Pharmacotherapy 24(10), 1358-1365 (2004).

9. M. Eckstein, "Cyanide as a chemical terrorism weapon," J. Emerg. Med. Serv. (JEMS) 29(8), suppl. 22-31 (2004).

10. B. Megarbane, A. Delahaye, D. Goldgran-Toledano, and F. J. Baud, "Antidotal treatment of cyanide poisoning," J. Chin. Med. Assoc. 66(4), 193-203 (2003).

11. I. Leybel, S. W. Borron, and C. J. Roldan, "Toxicity, cyanide," available at http://www.emedicine.com/emerg/topic118.htm (2006).

12. "FDA approves drug to treat cyanide poisoning," available at http:// www.fda.gov/bbs/topics/NEWS/2006/NEW01531.html (2006).

13. S. W. Borron, "Recognition and treatment of acute cyanide poisoning," J. Emerg. Nurs. 32(4 Suppl), S12-18 (2006).

14. S. W. Borron and F. J. Baud, "Acute cyanide poisoning: clinical spectrum, diagnosis, and treatment," Arh Hig Rada Toksikol 47(3), 307-322 (1996).

15. K. Alexander and S. I. Baskin, "The inhibition of cytochrome oxidase by diaminomaleonitrile," Biochim. Biophys. Acta 912(1), 41-47 (1987).

16. J. O. Egekeze and F. W. Oehme, "Cyanides and their toxicity: a literature review," Tijdschr Diergeneeskd 105(8), suppl. 2, 104-114 (1980).

17. M. Panda and N. C. Robinson, "Kinetics and mechanism for the binding of HCN to cytochrome c oxidase," Biochemistry 34(31), 10,009-10,018 (1995).

18. P. A. Lee, A. L. Sylvia, and C. A. Piantadosi, "Cyanide-related changes in cerebral $\mathrm{O}_{2}$ delivery and metabolism in fluorocarboncirculated rats," Toxicol. Appl. Pharmacol. 94(1), 34-44 (1988).

19. M. J. Koschel, "Management of the cyanide-poisoned patient," $J$. Emerg. Nurs. 32(4 Suppl.), S19-26 (2006).

20. J. Lee, J. Armstrong, K. Kreuter, B. J. Tromberg, and M. Brenner, "Non-invasive in vivo diffuse optical spectroscopy monitoring of cyanide poisoning in a rabbit model," Physiol. Meas 28(9), 1057-1066 (2007).

21. J. Lee, N. El-Abaddi, A. Duke, A. E. Cerussi, M. Brenner, and B. J. Tromberg, "Noninvasive in vivo monitoring of methemoglobin formation and reduction with broadband diffuse optical spectroscopy," J. Appl. Phys. 100(2), 615-622 (2006).

22. J. Lee, D. J. Saltzman, A. E. Cerussi, D. V. Gelfand, J. Milliken, T. Waddington, B. J. Tromberg, and M. Brenner, "Broadband diffuse optical spectroscopy measurement of hemoglobin concentration during hypovolemia in rabbits," Physiol. Meas 27(8), 757-767 (2006).

23. J. Lee, A. E. Cerussi, D. Saltzman, T. Waddington, B. J. Tromberg, and M. Brenner, "Hemoglobin measurement patterns during noninvasive diffuse optical spectroscopy monitoring of hypovolemic shock and fluid replacement," J. Biomed. Opt. 12(2), 024001 (2007).

24. F. Bevilacqua, A. J. Berger, A. E. Cerussi, D. Jakubowski, and B. J. Tromberg, "Broadband absorption spectroscopy in turbid media by combined frequency-domain and steady-state methods," Appl. Opt. 39(34), 6498-6507 (2000).

25. T. H. Pham, O. Coquoz, J. B. Fishikin, E. Anderson, and B. J. Tromberg, "Broad bandwidth frequency domain instrument for quantitative tissue optical spectroscopy," Rev. Sci. Instrum. 71, 2500-2513 (2000).

26. S. Merritt, G. Gulsen, G. Chiou, Y. Chu, C. Deng, A. EJ. Cerussi, A. J. Durkin, B. J. Tromberg, and O. Nalcioglu, "Comparison of water and lipid content measurements using diffuse optical spectroscopy and MRI in emulsion phantoms," Technol. Cancer Res. Treat. 2(6), 563-569 (2003). 
27. V. S. Sharma, R. B. Pilz, G. R. Boss, and D. Magde, "Reactions of nitric oxide with vitamin B12 and its precursor, cobinamide," Biochemistry 42(29), 8900-8908 (2003).

28. K. E. Broderick, P. Potluri, S. Zhuang, I. E. Scheffler, V. S. Sharma, R. B. Pilz, and G. R. Boss, "Cyanide detoxification by the cobalamin precursor cobinamide," Exp. Biol. Med. 231(5), 641-649 (2006).

29. K. E. Broderick, M. Balasubramanian, A. Chan, P. Potluri, J. Feala, D. D. Belke, A. McCulloch, V. S. Sharma, R. B. Pilz, T. D. Bigby, and G. R. Boss, "The cobalamin precursor cobinamide detoxifies nitroprusside-generated cyanide," Exp. Biol. Med. 232(6), 789-798 (2007).

30. G. C. Hayward, H. A. O. Hill, J. M. Pratt, N. J. Vanston, and R. J. P. Williams, "The chemistry of vitamin B(12). Part IV.1 The thermodynamic trans-effect," J. Chem. Soc. 6485-6493 (1965).

31. G. Guilbault and D. Kramer, "Ultrasensitive, specific method for cyanide using p-notrobenzaldehyde and o-dintrobenzene," Anal. Chem. 28, 834-836 (1966).

32. P. Renz, "Some intermediates in the biosynthesis of vitamin B12," Methods Enzymol. 18, 82-92 (1971).

33. W. G. Zijlistra, A. Buursma, and O. V. W. Assendelft, "Visible and Near Infrared Absorption Spectra of Human and Animal Hæmoglobin: Determination and Application: VSP," (2000).

34. P. Lundquist, H. Rosling, and B. Sorbo, "Determination of cyanide in whole blood, erythrocytes, and plasma," Clin. Chem. 31(4), 591-595 (1985).

35. J. G. Kim and H. Liu, "Investigation of biphasic tumor oxygen dy- namics induced by hyperoxic gas intervention: the dynamic phantom approach," Appl. Opt. 47(2), 242-252 (2008).

36. S. W. Borron, M. Stonerook, and F. Reid, "Efficacy of hydroxocobalamin for the treatment of acute cyanide poisoning in adult beagle dogs," Clin. Toxicol. 44(Suppl 1), 5-15 (2006).

37. J. B. Brierley, A. W. Brown, and J. Calverley, "Cyanide intoxication in the rat: physiological and neuropathological aspects," J. Neurol., Neurosurg. Psychiatry 39(2), 129-140 (1976).

38. J. B. Brierley, P. F. Prior, J. Calverley, and A. W. Brown, "Cyanide intoxication in Macaca mulatta. Physiological and neuropathological aspects," J. Neurol. Sci. 31(1), 133-157 (1977).

39. W. A. Groff Sr., F. W. Stemler, A. Kaminskis, H. L. Froehlich, and R. P. Johnson, "Plasma free cyanide and blood total cyanide: a rapid completely automated microdistillation assay," Clin. Toxicol. 23, 133-163 (1985).

40. F. J. Baud, P. Barriot, V. Toffis, B. Riou, E. Vicaut, Y. Lecarpentier, R. Bourdon,A. Astier, and C. Bismuth, "Elevated blood cyanide concentrations in victims of smoke inhalation," N. Engl. J. Med. 325(25), 1761-1766 (1991).

41. J. Lee, D. Mukai, K. Kreuter, S. Mahon, B. Tromberg, and M. Brenner, "Potential interference by hydroxocobalamin on cooximetry hemoglobin measurements during cyanide and smoke inhalation treatments," Ann. Emerg. Med. 49(6), 802-805 (2007).

42. Cyanokit package insert; available at http://www.doh.state.fl.us/ demo/ems/Cyanokit-FinalPI-15Dec152006.pdf, accessed Dec. 24, 2009 . 